\title{
Perspectives on Hepatic Metastases and the Minimally Invasive Approach to Resection
}

\begin{abstract}
Authors:
Elroy Patrick Weledji

Department of Surgery, Faculty of Health Sciences, University of Buea, Cameroon

Correspondence to elroypat@yahoo.co.uk

Disclosure: $\quad$ The author has declared no conflicts of interest.

Acknowledgements: The author acknowledges the acquisition of the Ronald Raven British Association of Surgical Oncologists (BASO) travel fellowship to the Hepatobiliary Unit, Aintree University Hospital, Liverpool, UK, which rendered the impetus behind this work.

Received:

27.07.20

Accepted:

27.04.21

Keywords:

Colorectal, liver metastases, minimally invasive surgery.

Citation:

EMJ Oncol. 2021;9[1]:53-61.

Abstract

Surgical resection is the most effective treatment approach in colorectal liver metastases. The improved survival in Stage IV colorectal cancer is associated with a better diagnosis and evaluation, proper decision-making, improved chemotherapy, and the adoption of parenchymal-sparing hepatic resections. Liver surgery was one of the last frontiers reached by minimally invasive surgery. Surgical techniques and specialised equipment evolved to overcome the technical limitations, making laparoscopic liver resections safe and feasible. The aetiology and pathophysiology of hepatic metastases are discussed along with the rationale for and efficacy of minimally invasive surgery for colorectal liver metastases. Improved imaging techniques, identification of genomic markers, advances in chemotherapy, and personalised therapy will further improve the outcome of minimally invasive surgery in the management of Stage IV colorectal cancer.
\end{abstract}

\section{INTRODUCTION}

\section{The Natural History of Hepatic Metastases}

The liver is the most common site for colorectal cancer (CRC) metastases, accounting for $80 \%$ of patients with Stage IV CRC and $40 \%$ as the only site of distant disease. Of the patients with CRC, $20-25 \%$ present with synchronous metastases and $50-60 \%$ will develop metachronous disease. Liver metastases develop in the absence of lymph node involvement and, presumably, this occurs via the haematogenous route (the portal circulation) in gastrointestinal tumours from where tumour

cells can embolise via the mesenteric veins. ${ }^{2}$ However, the fact that tumour cells from outside the gastrointestinal tract also commonly spread to the liver suggests that organ preference is not purely anatomical and the 'seed and soil' hypothesis, first proposed by Paget in $1889,{ }^{3}$ is still tenable. The complex tumour cell interactions that occur with the endothelial lining and lymphatic cells are, in part, what determines their final organ distribution. ${ }^{4}$ Tumour cells that invade lymphatics may also spread haematogenously via venolymphatic communications or directly via the thoracic duct. ${ }^{5}$ Some large metastases do not demonstrate spread to local periportal lymph nodes even in the presence of extensive disease within the liver. ${ }^{6}$ 
A liver metastasis may attain an enormous size, sometimes occupying much of the liver by concentric growth with extension in all directions, and may occasionally spread to adjacent structures such as the diaphragm by penetrating the usually unyielding Glisson's capsule.7 The right lobe of the liver is involved with metastases more frequently than the left lobe, although the reasons remain unclear as there is no gross difference of either arterial or portal blood received by each lobe. It may, however, be due to portal vein 'streaming', resulting in tumour emboli preferentially entering the right portal vein branches. ${ }^{8,9}$ Approximately one-third of patients with colorectal liver metastases (CRLM) cancer will have disease located in one lobe; 10 whereas multiple deposits throughout the liver are more commonly seen in patients with breast, oesophageal, gastric, and pancreatic cancer and are indicative of a more widespread metastatic process."

It has been estimated that that the subclinical phase of a liver metastasis (i.e., from a metastatic implantation to clinical appearance) may be 2.5-5 years. $^{12}$ This would suggest that survival rate may be improved if liver metastases are detected much earlier. A cluster of similar-sized metastases, suggestive of a common tumour embolic event clearly occurring in a segment or lobe, will leave the residual liver disease free; whereas metastases of differing sizes are probably indicative of showers of tumour emboli occurring at different times. ${ }^{1,12}$ Small lesions within the liver are usually asymptomatic and patients with advanced disease usually present with a combination of upper abdominal discomfort, weight loss, and general malaise. Pain may be due to the unremitting rapid growth of large metastases and is occasionally referred to the right shoulder, although central necrosis and infarction may also cause pain and pyrexia transiently. Hepatomegaly is indicative of advanced disease and may occasionally be accompanied by fulminant hepatic failure if the metastases are rapidly growing. Evidence of advanced liver failure such as jaundice, ascites, and occasionally portal hypertension are late signs and indicative of an extremely poor prognosis. ${ }^{7}$

In patients with carcinoid, the first presentation may be of carcinoid syndrome, characterised by diarrhoea, flushing, and wheezing due to excessive secretion of serotonin and tachykinin peptides from the hepatic metastases overwhelming its metabolism. The tumour, node, metastases (TNM) staging system does not adapt to recent advances in metastatic treatment. ${ }^{13-15}$ The survival of patients with resectable solitary metastasis (Stage IV disease) is better than patients with Stage II disease. . $3,14^{\text {Tumour deposits }}$ in adjacent vessels are associated with peritoneal disease, and tumour deposit with nodal disease (N2) has worse survival. ${ }^{14}$

\section{Liver Regeneration}

The ability of the surgeon to remove large volumes of liver tissue safely and with expectation of survival depends on a knowledge of the anatomy of the liver $^{16}$ and on an appreciation of the extraordinary rapid regenerative capacity following major resection, which have been extensively studied. ${ }^{17}$ Following resection as extensive as a right hepatectomy (at which half the liver mass is removed), liver size is regenerated within 3-4 weeks. This increase in size is accompanied by histological evidence of regenerative hyperplasia as early as 3 days after resection. During the period of liver regeneration liver function is depressed and the patient may require supportive measures. However, it is interesting to note that the outcome of regeneration following traumatic injury is different from liver regeneration following hepatocellular injury such as hepatitis, which follows the course of cirrhosis (alternating regeneration and fibrosis), dysplasia, and finally hepatocellular carcinoma after approximately 10-20 years. This implies that the mechanism and molecular pathways differ in the two modes of liver regeneration. ${ }^{18}$

\section{TREATMENT OF COLORECTAL LIVER METASTASES}

There is no advantage in delaying hepatic resection following diagnosis and patients should undergo liver resection as soon as is feasible. The old dogma that a waiting period is necessary to evaluate tumour aggressiveness is no longer tenable. The median survival of untreated CRLM following diagnosis is 6-12 months and 5 -year survival is extremely rare. ${ }^{19,20}$ For CRLM, $80 \%$ are initially non-resectable due to tumour size, location, and functional liver reserve.' 
Other factors that may indicate a poor outlook and exclude the possibility of a cure are the presence of abnormal liver function tests, spread of tumour to extrahepatic sites, and primary tumours that are not resected. ${ }^{7}$ Currently, patients with definitely unresectable disease may have widespread hepatic disease, non-resectable extrahepatic lesions, or multiple metastatic sites. ${ }^{1}$

In untreated patients, tumour burden is the major determinant of outcome and patients with solitary metastases usually live longer than those with multiple, bilobar disease. ${ }^{13} \mathrm{New}$ chemotherapy regimens including biologicals are bringing more patients to resection, including resectable extrahepatic disease. Resectability is the complete removal of liver metastases while leaving at least $30 \%$ of functional remnant liver. In several studies, metastases $>5 \mathrm{~cm}$ were associated with poorer survival than smaller metastases. ${ }^{8}$ Although larger liver metastases have usually been present for a longer time than smaller lesions, in the situation of a giant solitary metastasis the tumour biology may be such that the capacity for multiple metastases may well be limited and, therefore, the outcome may be good after resection. ${ }^{1}$

Although neoadjuvant chemotherapy such as folinic acid plus fluorouracil plus oxaliplatin (FOLFOX) as a first-line treatment and then single agent irinotecan as a second-line treatment has improved tumour response, the median survival for patients with unresectable disease is poor, and there is no 5-year survival. Resection, when feasible, confers a higher chance of cure and can improve 5-year survival to 34-60\%, 1,,21-23 However, apart from the risk of chemotherapy-associated steatohepatitis (CASH), the rationale in using neoadjuvant chemotherapy for patients with resectable disease has been supported by the better prognosis obtained compared to upfront surgery, due to the lower rate of positive surgical margins and the rendered ability to identify the subgroup of patients who will develop progressive disease while on chemotherapy.22,23

The resectability criteria for (CRLM) are expanded in an advanced multidisciplinary team (MDT) meeting alongside the evolution of imaging and neoadjuvant and adjuvant techniques such as thermal ablation, selective internal radiation therapy, and transarterial chemoembolisation. ${ }^{24-26}$ The management of Stage IV CRC would be optimised by bringing together all relevant specialties involved in colorectal metastatic disease management in a centralised highvolume centre. The major objective and endpoint of the advanced MDT meeting on Stage IV CRC management is resectability due to the impact on patient survival ( $40 \%>5$ years).,24,25 The main determinants of the decision-making process are the tumour statuses of both the primary tumour and metastases, the need for emergency surgery of a complicated primary tumour, and the resectability of both tumour sites..$^{24,25,27}$ The diagnosis and decision-making for the management of resectable, borderline resectable, or unresectable CRLM is expedited in the advanced MDT. The utilisation of protocols, appropriate preparation of patients, audit, and trial recruitments are optimised. Non-adherence to MDT decisions has been shown to result in a trend towards lower survival rates. ${ }^{28-30} \mathrm{~A}$ number of series with sufficient long-term follow-up indicate a 10-year survival after resection in 20$30 \%$ of patients. ${ }^{31,32}$

Unresectable unilobar disease may be treated by neoadjuvant chemotherapy followed by extended liver resection, with or without portal vein embolisation or associating liver partition and portal vein ligation for staged hepatectomy (ALPPS) ${ }^{33,34}$ to stimulate the size of the future liver remnant. For multiple bilobar CRLM, the strategies for improved margin clearance include staged resection, which entails a firststage local resection of metastases of the future left remnant liver followed by portal vein embolisation or ALPPS, and then a second-stage right hepatectomy 4 weeks later, after the left remnant has hypertrophied. ${ }^{1,24,25}$

Although high-quality contrast-enhanced CT and liver MRI are commonly used preoperatively, laparoscopic ultrasonography, usually performed with a high-resolution 7.5-10 $\mathrm{MHz}$ probe, allows for the direct visualisation of liver metastases in regard to segmental anatomy, local vascular involvement, and regional nodal disease. Laparoscopic ultrasonography improves the diagnostic accuracy of staging laparoscopy alone, provides additional information on resectability in $14-25 \%$ of patients, and detects occult metastases and new findings in $40-55 \%$ of cases. $^{25,35}$

Intra-operative ultrasound via real-time imaging aids planning at the time of resection and allows for the safe removal of all viable tumours, with a 
clear margin of $>1 \mathrm{~cm}$. It facilitates liver-sparing and microwave or radiofrequency thermal ablation techniques in patients with compromised parenchyma (CASH, prior liver resection), and avoids the 'small for size' syndrome. ${ }^{25,36}$

Anatomical liver resections follow anatomical planes and thus have better oncological clearance than non-anatomical liver resections. Major anatomical resections have better oncological clearance than limited segmental resections, with reduced recurrence rate and improved survival. However, segmental liver resection of localised tumours, based on Couinaud's liver segmental classification (Figure 1), would improve vascular control (less blood loss), minimise the risk of recurrence from intrahepatic spread, and reduce the amount of normal liver unnecessarily removed. ${ }^{16}$ Clearly, for small, awkwardly located lesions (such as the apex of Segment VIII in the axilla of the right and middle hepatic veins), local resection might be preferable to formal hemihepatectomy, whereby a whole, healthy lobe may need to be sacrificed for a small deposit. For larger metastases or multiple deposits, standard anatomical resections based on Couinaud segments should ensure adequate margins unless this increases the risk of postoperative liver failure.
Fortunately, secondary liver metastases from CRCs have better biology than metastases from other gastrointestinal sites and are amenable to non-anatomical surgical resections. In addition, a parenchymal-sparing approach in CRLM is supported by evidence that more aggressive resection at primary surgery does not prevent intrahepatic recurrence. ${ }^{37,38}$ Thus, the oncologically safe, non-anatomical, parenchymal-sparing resections are used for CRLM to achieve a complete metastasectomy. It is appropriately utilised in the modern setting of multimodal treatments and repeat resections. It may, however, result in compromising the vascularity of the adjacent residual liver tissue and may be technically more difficult with repeat resections. ${ }^{1,8,24,25}$

Simple wedge excision of peripheral lesions is not appropriate since it compromises the resection margin and risks the danger of leaving satellite metastases. ${ }^{1,24,25}$ A diligent search for other metastases should be carried out using intra-operative ultrasound before attempting to 'wedge out' an apparently superficial tumour nodule. $1,24,32$

Approximately $20 \%$ of patients have liver-only recurrence, with more than one-third occurring in the opposite side of the liver ${ }^{39}$ and hence amenable for re-resection. $124,25,40$

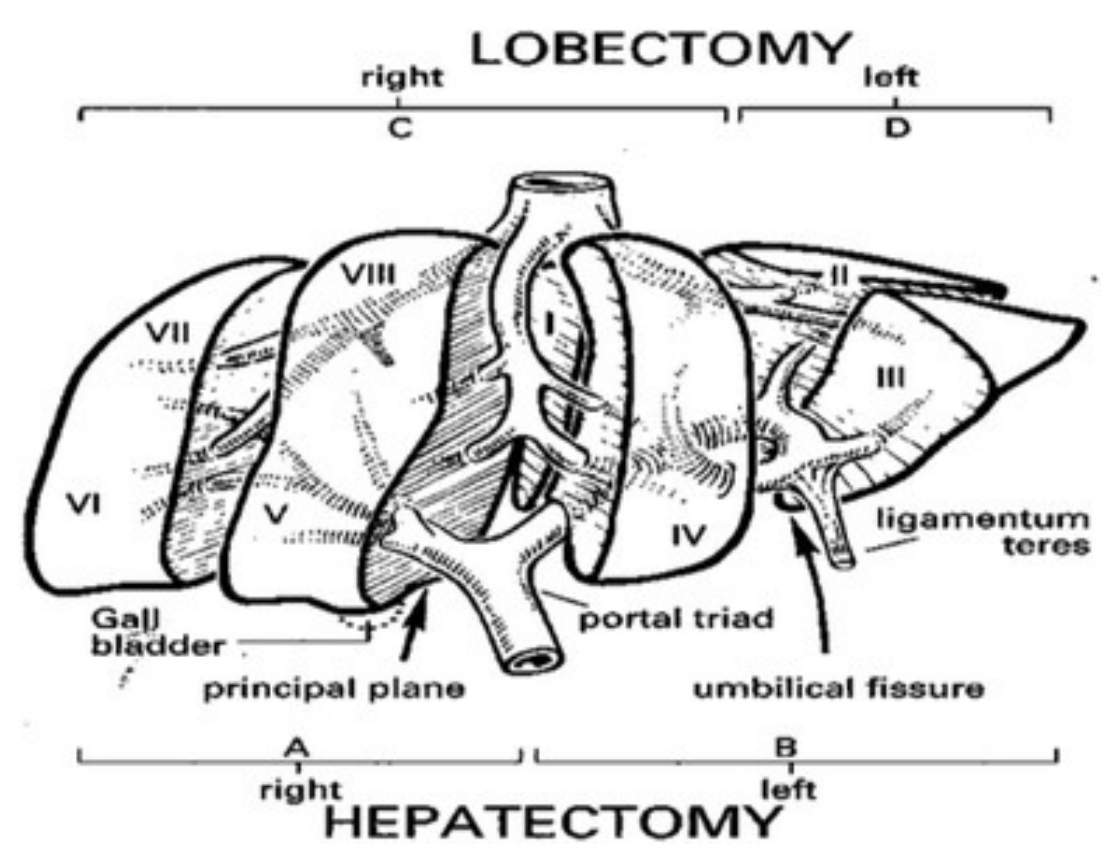

Figure 1: Couinaud's segmental anatomy of the liver (with permission: Weledji et al. Curr Surg Rep 2016;4:4).16 
When disease recurs in the liver, it is more often at some site distant from the original resection line and most likely to have arisen in undetected micrometastases present at the time of original liver resection., ${ }^{1,24}$

This would corroborate the importance of perioperative chemotherapy in surgical oncology as it increases progression-free survival. ${ }^{41}$ Thus, ideally, a resection margin of at least $1 \mathrm{~cm}$ should be attempted, judged by intraoperative ultrasonography, but if not technically possible narrow margins should not be an absolute contraindication to resection., ${ }^{1,24}$ There is controversy as to the significance of resection margin status following ablation with haemostatic devices as this will destroy the margin to some extent (1-3 mm) giving an appearance of a 'RO' margin (no tumour cells) in the patient remnant but an 'R1' margin (tumour cells present) in the pathological specimen.,24,25 Generally, the major determinant of success in the elderly ( $>80$ years of age) is the volume of residual liver (since liver adaptations following resection diminishes with age), and fitness for general anaesthesia. 1,8,24,25

\section{Minimally Invasive Surgery}

Laparoscopic surgery had been slowly introduced in surgical oncology because of the concern of inadequate margins or lymph node sampling, tumour seeding, missing small metastases, and poor pathological and oncological outcomes. The OSLO-COMET randomised controlled trial showed that in patients undergoing parenchymasparing liver resection for colorectal metastases, laparoscopic surgery was associated with significantly fewer postoperative complications compared to open surgery, was cost-effective, and the rate of free resection margins was the same. ${ }^{42}$ The LapOpHuva prospective, randomised controlled trial comparing laparoscopic liver resection (LLR) with open liver resection (OLR) in patients with CRLM showed LLR presenting with a lower global morbidity (11.5\% versus $23.7 \%$ ), but with similar severe complications. The long-term survival outcomes were similar in both groups. LLR involved more use of the Pringle manoeuvre (15.5\% versus $30.2 \%$ ) and a shorter hospital stay ( 4 versus 6 days). There were no differences regarding surgical time, blood losses, transfusion, and mortality. ${ }^{43}$ Thus, the study demonstrated that in selected patients with CRLM, LLR presented similar oncological outcomes to OLR, with the advantages of the short-term results associated with LLR.

The concerns of the rare air embolism are met by putting the patient in $15^{\circ}$ Trendelenberg position and careful surgical technique, especially when dissecting the hepatic veins.,24,44 In the current COVID-19 pandemic, just as with surgery during the HIV/AIDS epidemic, ${ }^{45}$ care should be taken during laparoscopy upon using disposable ports, with a vestibular flange to prevent splash-back and by deflating the abdomen prior to port withdrawal because any aerosol emanating from the port entry wound will harbour COVID-19.46,47 In addition to the currently advised personal protective equipment for healthcare staff in the operating theatre, this simple method would further lessen the risk of occupational transmission. Patients with COVID-19 would benefit from the reduced surgical stress of minimally invasive surgery, but it would be important to know the effect of immunosuppression from major LLR on COVID-19 disease progression. ${ }^{48}$ Larger resections, especially in patients with intrinsic liver disease, should be avoided if possible since postoperative COVID-19 infection might threaten the hypertrophic potential of the future liver remnant, placing the patient at risk of liver failurerelated death or insufficient hepatic reserve to survive any COVID-19-related complication. ${ }^{49}$

During the 1990s, minor resections of two or fewer easily accessible Couinaud's liver segments had been the standard of care. The posteriorsuperior segments (VII, VIII) and inferior segments (I, IVa) were excluded as they posed a higher surgical challenge from the extensive mobilisation required to bring those segments to the operative field. Resections of lesions located on anterolateral segments (II, III, IVb, V, VI) and left lateral sectionectomy (II, III) were performed systematically by laparoscopy in hepatobiliary centres. The posterior-superior resections had been indicated as 'major operations', despite including only two segments (VII, VIII). This was corroborated by the associated higher conversion rates, higher blood loss, prolonged operative times, and narrower surgical margins..$^{50}$ Resection of lesions located on posterior-superior segments and major liver resections were shown to be feasible but remain technically demanding and reserved for experienced surgeons in highvolume hepatobiliary centres. Laparoscopy- 
assisted and transthoracic port placement are useful strategies applied to difficult resections. ${ }^{44,50}$

In 2000, Cherqui et al..$^{51}$ published the feasibility study of LLR for both benign and malignant diseases of the liver including hepatocellular carcinoma in cirrhotic livers. Since then, nearly 10,000 minor and major LLRs as alternatives to open surgery have been reported in the literature, showing the wide acceptance and safety. ${ }^{48,52}$ Currently, the indications for LLR do not differ from those for open surgery. ${ }^{52,53}$ A recent metaanalytic study ${ }^{54}$ showed LLR as having a better peri-operative outcome than OLR for recurrent liver cancer, without compromising oncological outcome. With longer overall and median survival rates following recurrent resections, the indications for surgery are increasing with R1 surgery (complete tumour resection without safe margins) being justified for patients, with a response to preoperative chemotherapy.,12,25,28 It makes sense that minimally invasive procedures are made available to these elderly patients who may also have $\mathrm{CASH}$, prior liver resections, and other comorbidities. ${ }^{28,42,44}$

In addition, the majority of patients (approximately 65\%) develop intrahepatic recurrence within 3 years, even with the addition of systemic chemotherapy, but approximately $20 \%$ of these patients have liver-only recurrence, which may be suitable for re-resection.,24,25,31 Although repeat hepatectomy is often more difficult than the initial procedure because of dense adhesions and more friable and fibrotic liver parenchyma, ${ }^{55}$ reported mortality and morbidity rates after repeat liver resection of metastases are surprisingly similar to those reported after initial hepatectomy. ${ }^{35,56}$ Adjunctive treatment such as laparoscopic radiofrequency or microwave ablation is acceptable for patients of high surgical risk for liver resection or with small solitary CRLM.1,24,25 Therefore, the favourable biology of CRLM have enabled patients to live with their disease with repeat resections for recurrence. ${ }^{1,24,25,31}$

However, oncogenic mutations of RAS genes (NRAS and NRAS) controlling cell proliferation have been associated with worse disease-free and overall survival following CRLM resection, even with adjuvant anti-epidermal growth factor cetuximab therapy. ${ }^{57}$ The addition of cetuximab to FOLFOX in the neoadjuvant setting results in an overall survival advantage in patients with advanced disease who have the KRAS exon 2 wild-type tumour genotype. ${ }^{58}$ Thus, the rationale for neoadjuvant chemotherapy, even for resectable lesions, and the addition of biologic agents for the KRAS exon 2 wild-type is to destroy occult micrometastases and increase progression-free survival. ${ }^{1,23,24}$

After resection of the primary CRC, neoadjuvant chemotherapy with mFOLFOX6 and the vascular endothelial growth factor inhibitor bevacizumab for patients with resectable synchronous CRLM was safe and feasible with an impressive response rate of $72.9 \%$ and $90.9 \%$ of patients proceeding to liver resection; ${ }^{59}$ however, it lacked proven benefit as adjuvant treatment. 60 Where CRLM are unresectable, chemotherapy may downsize tumours and improve biological selection for resection. This is seen as a complete radiological response, which depends on the quality and completeness of preoperative imaging, or as '“missing' metastases. As a complete radiological response does not signify a complete pathological response, liver resection of curative intent would include all initial and currently known sites of disease (Figures 2 and 3), ${ }^{1,24}$ Roboticassisted resections are feasible as demonstrated in reported case series. The 3D view and greater range of movement can be useful for complex resections. ${ }^{61}$ The dynamic applicability of the 3D planning to navigation during operation may also improve operative results. ${ }^{62}$

\section{One Stage: Simultaneous or Staged Procedure?}

The decision as to whether the operations for the primary tumour and liver metastases are performed at the same time (simultaneous) or separately (staged) is made at the advanced MDT meeting and in discussions with the patient. The advantages of a one-stage (simultaneous) operation ${ }^{63,64}$ are the decreased risk of disease dissemination (transperitoneally), no repeated postoperative immunosuppression causing increased tumour growth, and lower costs. A staged procedure would allow for the assessment of biological behaviour of metastases, avoid operating on patients who are progressing while on chemotherapy, and allow more precise selection for curative surgery.1,24,65 Delayed hepatic resection may not impair survival but help to select those patients most likely to benefit 


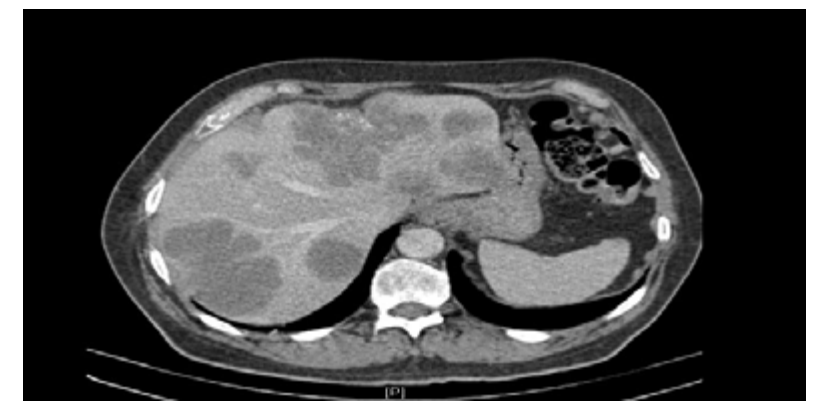

Figure 2: Pre-operative chemotherapy CT scan of colorectal liver metastases (with consent).

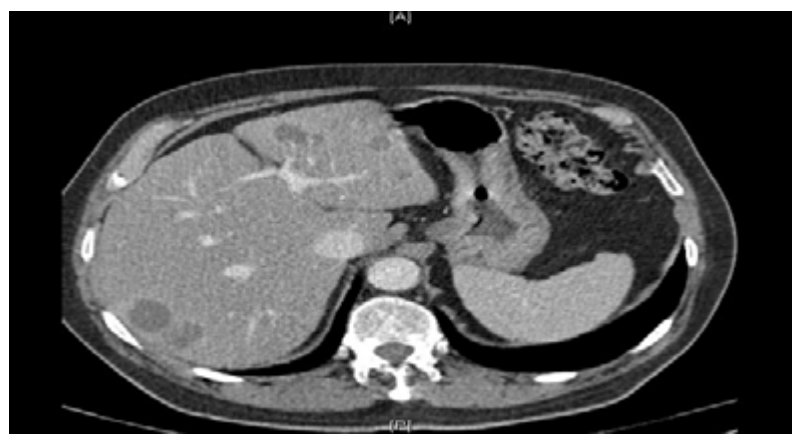

Figure 3: CT scan post-chemotherapy of colorectal liver metastases (with consent).

from hepatic resection (i.e., stable disease). ${ }^{66,67}$

For mid- and low-rectal primary tumours, chemoradiotherapy is often needed and, in addition to a difficult resection, a one-stage surgery is not recommended.,24,25 One-stage surgery is not advocated for complex colonic and upper-rectal primary tumours, for highrisk patients, or when hepatectomy is major ( $>3$ segments). Minor liver resections (2 segments or fewer) may be safely performed at the same time as colorectal resection (open or laparoscopic) when both the primary tumour and the metastases are easily resectable. The outcomes are similar to sequential surgery in this scenario., ${ }^{1,24,68}$

\section{The Four Clinical Scenarios of Stage IV Colorectal Cancer}

The management of the four clinical scenarios are as follows:

> For asymptomatic CRC and resectable synchronous CRLM, chemotherapy is first, with or without radiotherapy, for rectal cancer. It is followed either by surgery in a one-stage procedure for patients with limited hepatic disease and easy to resect primary tumour, or by staged (liver-first) surgery for other patients.

> For asymptomatic CRC and non-resectable synchronous CRLM, the consensus is for optimal chemotherapy first, with the aim of making the liver metastases resectable. This is followed by hepatic surgery and then resection of the primary tumour.

> For symptomatic CRC and resectable synchronous CRLM, recommendations are for resection of the primary tumour for perforated or occlusive tumours (but not for tumours with bleeding causing anaemia), followed by chemotherapy and then surgery for liver metastases.

> For symptomatic CRC and non-resectable synchronous CRLM, recommendations are for resection of the primary tumour for perforated or occlusive tumours, followed by chemotherapy and then surgery for liver metastases if tumour shrinkage is achieved. For tumours with bleeding causing anaemia, induction chemotherapy is recommended 
to down-size both the primary tumour and livermetastases, followed by surgery at the site with the most significant tumour load, which is usually the liver (i.e., a reverse approach). ${ }^{1,24,25}$

Thus, although the treatment strategy depends on the clinical scenario, ${ }^{69}$ the disease being systemic, and synchronous disease, which has been widely recognised as prognostically unfavourable in various patient cohorts, chemotherapy should come before surgery in most cases. ${ }^{70,71}$

\section{CONCLUSIONS}

Both the proper selection of patients who will benefit from liver resection and a high degree of experience in minimally invasive surgery are warranted in a hepatobiliary unit. Improved imaging techniques, identification of genomic markers, advances in chemotherapy, and personalised therapy will further improve the outcome of minimally invasive surgery in the management of Stage IV CRC.

\section{References}

1. Jones RP et al. Defined criteria for respectability improves rates of secondary resection after systemic therapy for liver limited metastatic colorectal cancer. Eur J Cancer. 2014;50(9):1590-601.

2. Fisher ER, Turnbull RB. The cytological demonstration and significance of tumor cells in the mesenteric venous blood in patients with colorectal carcinoma. Surg Gynecol Obstet. 1955;100(1):102-8.

3. Paget S. The distribution of secondary growths in cancer of the breast. Lancet. 1889;8(2):571-3.

4. Naito $\mathrm{S}$ et al. Correlation between the in vitro interaction of tumor cells with an organ environment and metastatic behavior in vivo. Invasion Metastasis. 1987;7(1):16-29.

5. Fisher B, Fisher ER. The interrelationship of haematogenous and lymphatic tumour cell dissemination. Surg Gynecol Obstet. 1966;122(4):791-8.

6. Dworkin MJ et al. Importance of hepatic artery node involvement in patients with colorectal liver metastases. J Clin Pathol. 1995;48(3):270-2.

7. Foster JH, Lundy J. Liver metastases. Curr Probl Surg. 1981;18(3):157-202.

8. Scheele $\mathrm{J}$ et al. Indicators of prognosis after hepatic resection for colorectal secondaries. Surgery. 1991;110(1):13-29.

9. Holbrook RF et al. Patterns of colorectal liver metastases according to Couinaud's segments. Dis Colon Rectum. 1995:38(3):245-8.

10. Cady B, Stone MD. The role of surgical resection of liver metastases in colorectal carcinoma. Semin Oncol. 1991;18(4):399-406

11. Pickren JW et al., "Liver metastasis: analysis of autopsy data," Weiss L, Gilbert HA (eds.), Liver Metastasis: A Monograph Series (1982) Boston: GK Hall Medical Publishers, pp.2-19.20
12. Finlay IG et al. Growth rate of hepatic metastases in colorectal carcinoma. $\mathrm{Br} J$ Surg. 1988;75(7):641-4

13. Gayowski TJ et al. Experience in hepatic resection for metastatic colorectal cancer-analysis of clinical and pathological risk factors. Surgery. 1994;116(4):703-11

14. Nagtegal ID, Quirke P. Colorectal tumour deposits in the mesorectum and pericolon: a critical review. Histopathology. 2007;51(2):141-9.

15. Poston GJ et al. Urgent need for a new staging system in advanced colorectal cancer. J Clin Oncol. 2008;26(29):4828-33

16. Weledji EP, Ngounou E. The impact of segmental anatomy on hepatic oncologic resections. Curr Surg Rep. 2016;4:4.

17. Blumgart $\mathrm{LH}$ et al. Observations on liver regeneration after right hepatic lobectomy. Gut. 1971;12(11):922-8.

18. Ramakrishna $\mathrm{G}$ et al. From cirrhosis to hepatocellular carcinoma: new molecular insight on inflammation and cellular senescence. Liver Cancer. 2013;2(3):357-63.

19. Lykoudis PM et al. Systematic review of surgical management of synchronous liver metastases. $\mathrm{Br} \mathrm{J}$ Surg. 2014;101(6):605-12.

20. Manfredi S et al. Epidemiology and management of liver metastases from colorectal cancer. Ann Surg. 2006;244(2):254-9.

21. August DA et al. Lymphatic spread of tumour to extrahepatic sitesimplications for the follow-up and treatment of patients with colorectal cancer. Cancer. 1985;55(7):1490-4.

22. Adson MA et al. Resection of hepatic metastases from colorectal cancer. Arch Surg. 1984;119(6):647-51.

23. Finlay IG, McArdle CS. Occult hepatic metastases in colorectal carcinoma. $\mathrm{Br} J$ Surg. 1986:73(9):732-5.

24. Adam R et al. Managing synchronous liver metastases from colorectal cancer: multidisciplinary international consensus. Cancer Treat Res. 2015:41(9):729-41.

25. Weledji EP. Centralization of liver cancer surgery and impact on multidisciplinary teams working on stage IV colorectal cancer. Oncol Rev. 2017;11(2):331.

26. Gruber-Rouh T et al. Current strategies in interventional oncology of colorectal liver metastases. Br J Radiol. 2016;89:(1064)20151060.

27. Wanebo $\mathrm{HJ}$ et al. Current perspectives on repeat hepatic resection for colorectal carcinoma: a review. Surgery. 1996;119(4):361-71.

28. Goyer $\mathrm{P}$ et al. Single-center multidisciplinary management of patients with colorectal cancer and resectable synchronous liver metastases improves outcomes. Clin Res Hepatol Gastroenterol. 2013;37(1):47-55.

29. Viganò I et al. Colorectal cancer with synchronous resectable liver metastases: monocentric management in a hepatobiliary referral centre improves survival outcomes. Ann Surg Oncol. 2015;22:1533-9.

30. Vasudevan SP et al. Close collaboration between local and specialist multidisciplinary teams allows 'fast-tracking' of patients with colorectal liver metastases. Colorectal Dis. 2013;15(10):1253-56

31. Lordan JT et al. A 10-year study of outcome following hepatic resection for colorectal liver metastasesthe effect of evaluation in a multidisciplinary team setting. Eur $J$ Surg Oncol. 2009;35(3):302-6.

32. Prades $\mathrm{E}$ et al. Is it worth reorganizing cancer services on the basis of multidisciplinary teams (MDTs)? A systematic review of the objectives and organization of MDTs and their impact on patient outcomes. Health Policy. 
$2015 ; 119(4): 464-74$

33. May BJ, Madoff DC. Portal vein embolization: rationale, technique, and current application. Semin Intervent Radiol. 2012;29(2):81-9.

34. Zhang GQ et al. Associating liver partition and portal vein ligation for stage hepatectomy (ALPPS): a new strategy to increase liver surgical resection. Int J Surg. 2014;12(5);437-41.

35. Hoch $\mathrm{G}$ et al. Is intraoperative ultrasound still useful for the detection of colorectal cancer liver metastases? HPB (Oxford). 2015;17(6):514-9

36. Kingham TP et al. Hepatic parenchymal preservation surgery: decreasing morbidity and mortality rates in 4,152 resections for malignancy. J Am Coll Surg. 2015;2020(4):471-9.

37. Kokudo $\mathrm{N}$ et al. Anatomical major resection versus non-anatomical limited resection for liver metastases from colorectal carcinoma. Am J Surg. 2001;181(2):153-9.

38. Mise $Y$ et al. Parenchymal-sparing hepatectomy in colorectal liver metastasis improves salvageability and survival. Ann Surg. 2016;263(1):146-52.

39. Nordlinger B et al., "Surgical resection of hepatic metastases: multicentric retrospective study by the French Association of Surgery," Nordlinger B, Jaeck D (eds.), Treatment of Hepatic Metastases of Colorectal Cancer (1992) Paris: Springer-Verlag, pp.129-61.

40. Adam $\mathrm{R}$ et al. Liver resection for colorectal metastases: the third hepatectomy. Ann Surg. 2003;238(6):871-83.

41. Fisher B. Biological research in the evolution of cancer surgery: a personal perspective. Cancer Res. 2008;68(24):10007-20.

42. Fretland AA et al. Laparoscopic versus open resection for colorecta liver metastases: the OSLO-COMET randomized controlled trial. Ann Surg. 2018;267(2):199-207.

43. Robles-Campos $\mathrm{R}$ et al. Open versus minimally invasive liver surgery for colorectal liver metastases (LapOpHuva): a prospective randomized controlled trial. Surg Endosc. 2019;33(12):3926-36.

44. Schiffman SC et al. Laparoscopic versus open liver resection for metastatic colorectal cancer: a metaanalysis of 610 patients. Surgery. 2015:157(2):211-22

45. Weledji EP et al. Gastrointestinal surgery and the acquired immune deficiency syndrome. Ann Med Surg (Lond). 2015;4(1):36-40.

46. Hanbali $\mathrm{N}$ et al. A safe method to evacuate pneumoperitoneum during laparoscopic surgery in suspected COVID-19 patients. Annals R Coll Surg Engl. 2020;102(5):392-3.

47. Doremalen $\mathrm{N}$ et al. Aerosol and surface stability of SARS-CoV-2 as compared with SARS-CoV-1. N Eng J Med. 2020;382(16):1564-7.

48. Weledji EP. Cytokines and the metabolic response to surgery. J Clin Cell Immunol. 2014;5:2.

49. Tzenga $D$ et al. Surgical decisionmaking and prioritization for cancer patients at the onset of the COVID-19 pandemic: a multidisciplinary approach. Surg Oncol. 2020;34:182-5.

50. Coelho FT et al. Laparoscopic liver resection: experience based guidelines. World J Gastrointest Surg. 2016;8(11):5-26.

51. Cherqui D et al. Laparoscopic liver resections: a feasibility study in 30 patients. Ann Surg. 2000;232(6):753-62.

52. Wakabayashi $\mathrm{G}$ et al. Recommendations for laparoscopic liver resection: a report from the second international conference held in Morioka. Ann Surg. 2015;261(4):619-29.

53. Garden OJ et al. Guidelines for resection of colorectal cancer liver metastases. Gut. 2006;55(Suppl 3):iii1-8.

54. Liang $Y L$ et al. Perioperative outcomes comparing laparoscopic with open repeat liver resection for post-hepatectomy recurrent liver cancer: a systematic review and metaanalysis. Int J Surg. 2020;79:17-28.

55. Elias D et al. Repeat hepatectomy for cancer. Br J Surg. 1993;80(12):1557-62.

56. Jones RP, Poston G. Resection of liver metastases in colorectal cancer in the era of expanding systemic therapy. Annu Rev Med. 2017;68:183-96.

57. Nortlinger $B$ et al. Combination of surgery and chemotherapy and the role of targeted agents in the treatment of patients with colorectal liver metastases: recommendations from an expert panel. Ann Oncol. 2009;20(6):985-92

58. Primrose $\mathrm{J}$ et al. A randomized clinical trial of chemotherapy compared to chemotherapy in combination with cetuximab in k-RAS wild type patients with operable metastases from colorectal cancer: the new EPOC study. J Clin Oncol. 2013;31(15): 3504.
59. Jones RP et al. Colorectal liver metastases: a critical review of state of the art. Liver Cancer. 2016;6(1):66-71.

60. Nordlinger B et al. Should the results of the new EPOC trial change practice in the management of patients with resectable metastatic colorectal cancer confined to the liver? J Clin Oncol. 2015;33(3):241-3.

61. Montali R et al. Robotic versus laparoscopic hepatectomy: what is the best minimally invasive approach? Ann Surg. 2015;262(2):e70

62. Hallet $\mathrm{J}$ et al. Systematic review of the use of pre-operative simulation and navigation for hepatectomy: current status and future perspectives. J Hepatobiliary Pancreat Sci. 2015;22(5):353-62.

63. Feng $Q$ et al. Timing of hepatectomy for resectable synchronous colorectal liver metastases: for whom simultaneous resection is more suitable - a meta-analysis. PLos One. 2014;9(8):e104348.

64. Slesser AA et al. Outcome of simultaneous resections for patients with synchronous colorectal liver metastases. Eur J Surg Oncol. 2013;39(12):1384-93.

65. Reddy SK et al. Simultaneous resection of colorectal cancer and synchronous liver metastases: a multi-institutional analysis. Ann Surg Oncol. 2002;14(12):3481-91.

66. Lambert LA et al. Interval hepatic resection of colorectal metastases improves patient selection. Arch Surg. 2000;135(4):473-9.

67. Yin $Z$ et al. Timing of hepatectomy in resectable synchronous colorectal liver metastases (SCRLM): simultaneous or delayed? Hepatology. 2013;57(6):2346-57.

68. Tanaka K et al. Outcome after simultaneous colorectal and hepatic resection for colorectal cancer with synchronous metastases. Surgery. 2004;136(3):650-9.

69. Chow FC, Chok KS. Colorectal liver metastases: an update on multidisciplinary approach. World J Hepatol. 2019;11(2):150-72.

70. Araujo R et al. Comparison between perioperative and postoperative chemotherapy after potentially curative hepatic resection for metastatic colorectal cancer. Ann Surg Oncol. 2013;20(13):4312-21.

71. Lehmann $\mathrm{K}$ et al. Chemotherapy before liver resection of colorectal metastases: friend or foe? Ann Surg. 2012;255(2):237-47. 